\title{
The identity of certain representation algebra decompositions
}

\author{
S. B. Conlon and W. D. Wallis
}

Let $G$ be a finite group and $F$ a complete local noetherian commutative ring with residue field $\bar{F}$ of characteristic $p \neq 0$. Let $A(G)$ denote the representation algebra of $G$ with respect to $F$. This is a linear algebra over the complex field whose basis elements are the isomorphism-classes of indecomposable finitely generated FG-representation modules, with addition and multiplication induced by direct sum and tensor product respectively. The two authors have separately found decompositions of $A(G)$ as direct sums of subalgebras. In this note we show that the decompositions in one case have a cormon refinement given in the other's paper.

We adopt the notation of [2]; suppose then that $H$ is a normal p-subgroup of $G$ and $H \leq R \leq G$. We shall show that the decompositions (25) and (26) of [2] have a common refinement given in Proposition 5.5 of [1]. The problem will be resolved by showing that the idempotents $E_{S}$ introduced in [2, p. 400] are the sums of idempotents $u_{S}$ giving the decomposition 5.5 of [1]. As the $E_{S}$ are linear combinations of the $I_{R}$ of $[2, \mathrm{p} .398]$ it will be sufficient to show the same for the $I_{R}$. The idempotents $F_{K}$ mentioned in (27) of [2, p. 401] have been discussed in [1] and have been shown there to be sums of the $u_{S}$ 's already. The intersection of the decompositions given by the $E_{S}$ and the $F_{K}$ in (27) 
of [2] is similarly refined by 5.5 of [1].

We recall that $P(G)$ is the projective ideal of $A(G)$ and $P^{G}(R)=(P(R))^{G}$, that is, the image of $P(R)$ in $P(G)$ under the induction map ind ${ }_{R \rightarrow G} \cdot P^{G}(R)$ is an ideal of $P(G)$. All the idempotents $I_{R}$ can be considered to lie in $P(G / H)$ and so we may without loss of generality assume that $H$ is the trivial subgroup of $G$. $I_{R}$ is then the idempotent generator of $P^{G}(R)$.

From Proposition 5.11 of [1], we have that $I_{G} \in \Sigma_{R} P^{G}(R)$, where the sum runs over a complete set $Y$ of non-conjugate $p^{\prime}$-cyclic subgroups $R$ of $G$. For $R \in Y$, write $P^{\prime} G(R)=\Sigma_{R^{\prime}} P^{G}\left(R^{\prime}\right)$, the sum being over those $R^{\prime} \in Y$ which, to within conjugacy in $G$, are properly contained in $R$. Using the Mackay formula for the tensor product of induced representations, we see that the element $u_{R}=(1 /|N(R): R|) \quad\left\{\left(I_{R}\right)^{G}\right\}$ of $A(G)$ is the identity of $P^{G}(R)$ modulo $P^{\prime G}(R)$. Using induction on $|R|, R \in Y$, we see that each $I_{R}$ is a combination of the $u_{S}^{\prime}$ 's, as required.

\section{References}

[1] S.B. Conlon, "Decompositions induced from the Burnside algebra", $J$. AZgebra 10 (1968), 102-122.

[2] W.D. Wallis, "Decomposition of representation algebras", J. Austral. Math. Soc. 10 (1969), 395-402.

University of Sydney, Sydney, New South Wales, and University of Newcastle, Newcastle, New South Wales. 